\title{
Scientific Rationale, Clinical Protocols, and Positive Patient Outcomes of the EPIC Technique Spinal Procedure
}

\author{
Stan Pierce Jr DC* \\ New technology, Sherman College of Chiropractic, Life University, USA
}

Submission: February 16, 2021; Published: March 01, 2021

*Corresponding author: Stan Pierce Jr DC, New technology, Sherman College of Chiropractic, Life University, Florida, USA

Abstract

Inside the chiropractic profession, there are many diverse procedures and approaches to spinal care. Although each individual Doctor of Chiropractic (DC) may practice chiropractic with their own individual systems and techniques of care, those procedures are rooted in established techniques taught in the curriculum of various chiropractic colleges and universities. One of those established techniques is called EPIC. "EPIC" is an acronym for Evolutionary Percussive Instrument Corrections. It is a chiropractic technique that focuses on the use of progressively advancing diagnostic and adjusting technology to correct the craniocervical junction alignment. Its mission statement is: "We believe in challenging the status quo in healthcare, as well as in chiropractic. Forever striving to improve our quality of care, we will always look for the next conceptual or technological breakthrough that will help us achieve better spinal corrections for optimal patient health."

\section{History}

The EPIC technique is a direct genealogical descendant of the technique originally developed by the founding family of chiropractic. In 1895, Daniel David “DD” Palmer, DC first discovered and termed the spinal "subluxation", defined as a condition of the spine where a vertebra has lost its correct juxtaposition to the extent of disrupting the nervous system function. His son, Bartlett Joshua "BJ" Palmer, DC spent a lifetime of chiropractic research and concluded the premise of ADIO (Above-Down-Inside-Out) in referencing the flow of the nervous system primarily going from the brain, down the spinal cord, out the nerves to the effected/ controlled areas. Based upon this concept, Dr. BJ Palmer focused on refining a procedure to analyze and adjust the 2 upper cervical vertebral misalignments calling that area "the true subluxation" and, in the early 1900s, developed a technique called HoleIn-One (HIO)/Toggle focusing on adjusting this unique area. The existence of the subluxation's neurologic imbalance was determined through neurophysical examination. The direction of atlas (C1) malposition was then obtained from radiographic evaluation and was defined through positional listings (i.e. ASLP, AIRP, etc.) to give a general understanding of what direction the chiropractor needed to adjust it. The axis (C2) malposition was given a separate general designation from the radiographs and required a separate adjustment. Following the adjustment, the neurophysical criteria were re-examined to assess change, but post adjustment radiographs were not standardized to be taken. His approach was the foundation of the science, results, and public perspective of the beginning of the chiropractic profession and was called the Toggle technique.

In the 1930s, John Grostic, DC, refined the radiographs and measured angles used to assess the cervical malposition, quantified these malpositioned vertebrae on a mathematical engineering platform. He refined the adjusting art to reduce the amount and depth of thrust, and to improve the accuracy of the adjusting line of drive. He utilized a post x-ray analytical comparison after the first adjustment to objectively verify alignment had been improved. This technique was known as the Grostic technique.

In the 1970s, Roy Sweat, DC, developed a 2-axis, tablemounted, adjusting instrument that could deliver a percussive soundwave to align the upper cervical spine. He refined the line of drive measurement into a degree-specific calibrated adjustment, still using post adjustment examination and x-ray verification. Although the C1 vertebra was measurably misaligning around the $\mathrm{Z}$ and $\mathrm{Y}$ axis of the right-handed Cartesian coordinate system, Dr. Sweat's instrument was designed to pivot around the patient's Z 
and $\mathrm{X}$ axis with a resultant $\mathrm{Y}$ directional effect. This technique has been known as the Atlas Orthogonal technique.

In 2001, Stan Pierce Sr., DC, and Stan Pierce Jr., DC, added further refinements to the radiographic analysis protocol to include data transposition between radiographs for anatomical clarity, methods to validate reference line determination and measurement accuracy, and evaluation methods to determine genetic abnormalities that would affect the understanding of each patient's "normal" position into which they needed to be adjusted. Their chiropractic technique was the first to upgrade to digital x-ray with computerized analysis. Additionally, they created a more refined patient positioning protocol to control biomechanical reductions of the adjustment, improved accuracy of the instrument's line of drive vector calibration and developed a comprehensive post $\mathrm{x}$-ray interpretation to improve case management. This technique was known as the Advanced Orthogonal technique.

In 2014, Stan Pierce Jr., DC, launched a more aggressive evolution of developments in this lineage of concepts and technology. Guiding the same board of directors of his previous technique, Dr. Pierce Jr. lead a team to streamline imaging analysis, remove analysis error through advanced software coding, identify additional genetic considerations, and refine anatomical considerations. They then enhanced biomechanical influences in patient positioning through eliminating error in skull positioning, and other errors in the vector calibration. His team created the first 3-axis percussive adjusting instrument thus becoming the only technique in chiropractic that can purely calibrate the percussive adjusting force to match the axis of vertebral malposition. They developed a digital calibrating system and laser targeting system for the adjustment. His team developed a software program to assist the doctor in clinical implementation of performing the biomechanical influences and vector-calibrating accuracy, and to obtain and compare clinical data on an international platform for standardizing quality of care, also producing a vast amount of data points for clinical studies. This technique is known as Evolutionary Percussive Instrument Corrections (EPIC) and is taught by Dr. Pierce Jr. and his team in numerous chiropractic institutions of higher education.

\section{Neurological Effect}

The balance of the body's nervous system is dramatically affected by the relationship of the craniocervical junction. Much of the balance of the body is determined through neurologic control called mechanoreceptor feedback loops. Mechanoreceptor feedback is given in large part by the spindles of the muscles, and the muscle with the highest density of spindles per gram of tissue is the obliquus capitis inferioris muscle (242 spindles/gram of tissue) that inserts at the transverse process of the C1 vertebra and the spinous process of the C2 vertebra. The second most dense muscle is the obliquus capitis superioris with 190 spindles/ gram of tissue. For comparison, the lumbar erector muscles have 40 spindles/gram of tissue (Figure 1).

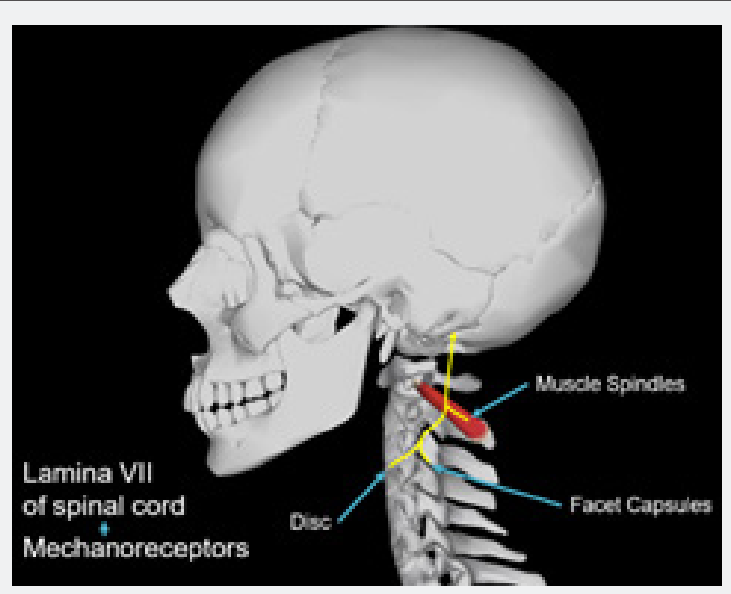

Figure 1

When the left and right obliquus capitis inferioris muscles go out of tonal balance due to craniocervical subluxation, this can disrupt the bilateral balance of spindle mechanoreceptor neurofeedback to the cerebellum. The cerebellum then transmits the imbalance to the vestibular nucleus, and in response, the vestibular nucleus may stimulate a neurologic alteration of the postural spinal muscle balance. Additionally, disrupted mechanoreceptor feedbackto thecerebellum may transmit through to the limbic, sensory, and cerebral cortices. It is plausible that a malposition of the craniocervical junction could affect emotional stability, dermatomal distributions, hypersensitivities, cognitive functions, memory and more through this neurodysfunction. In that the cortex directly communicates with the hypothalamus, and the hypothalamus controls the pituitary glands endocrine regulation, it is plausible that the neurodysfunction of the craniocervical junction could affect the regulation of hormones. Additionally, the hypothalamus communicates through the pons, to the sympathetic chain, to the intermediate lateral tracts of the spinal cord which can then affect immune function, blood vessel dilation, annulus of the vertebral discs, viscera of the organs, etc. 


\section{Orthopedics and Rheumatology Open Access Journal (OROAJ)}

Thus, it could be theorized that a craniocervical subluxation may contribute to an extremely wide variety of clinical conditions and associated symptomatology, as well as alter the overall health of the patient (Figure 2).

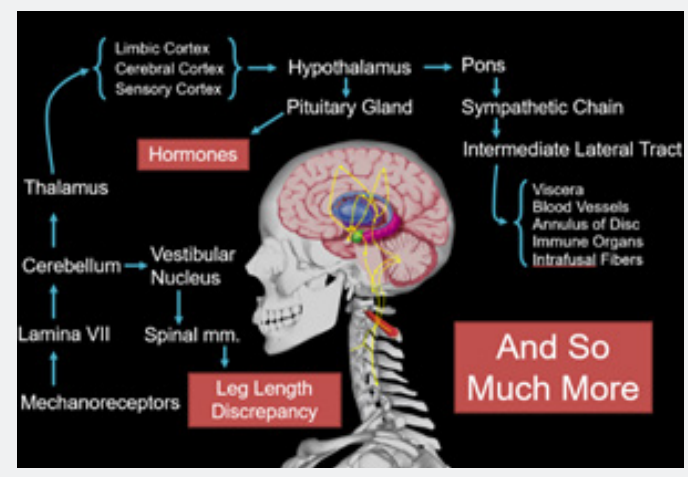

Figure 2

\section{Vascular Effect - Arterial}

Like all muscles, the myotonicity of the arterial walls is neurologically controlled. It is possible that an alteration in the above referenced mechanoreceptor feedback loop through the intermediate lateral (IML) tract to the myotonicity of the blood vessels could alter the diameter size of the vessel and the associated rate of blood flow. Altered diameter and flow rate may result in altered blood pressure. This is a plausible explanation for the documented patient outcomes of significantly reduced high blood pressure from a craniocervical chiropractic alignment procedure referenced in the study below.

Study: Atlas vertebra realignment and achievement of arterial pressure goal in hypertensive patients: a pilot study Journal of Human Hypertension. 2007 G. Bakris, M. Dickholtz Sr, et al. [1] "Anatomical abnormalities of the cervical spine at the level of the Atlas vertebra are associated with relative ischemia of the brainstem circulation and increased blood pressure (BP). This study indicated that restoration of Atlas alignment is associated with marked and sustained reductions in BP like the use of twodrug combination therapy."

Another potential arterial effect of a craniocervical subluxation is a directional alteration in the structural pathway of the vertebral artery. Termed the "vertebral" artery due to its ascension through the transverse foramen of the cervical vertebrae, the vertebral artery takes four right angle turns as it passes through the craniocervical junction. It is plausible that a rotationally misaligned C1 (atlas) vertebra may cause an abnormal torsion of the vertebral artery and resultingly affect flow rate (Figure 3). Additionally, a craniocervical subluxation that includes a rotationally displaced C1 (atlas) vertebra and a counterrotationally displaced C2 (axis) vertebra can potentially cause additional torsion to the vertebral artery. In that the vertebral artery is the primary blood supply to the midbrain and cranial nerve centers, it is plausible that a craniocervical subluxation could alter the vascular supply to the brain.

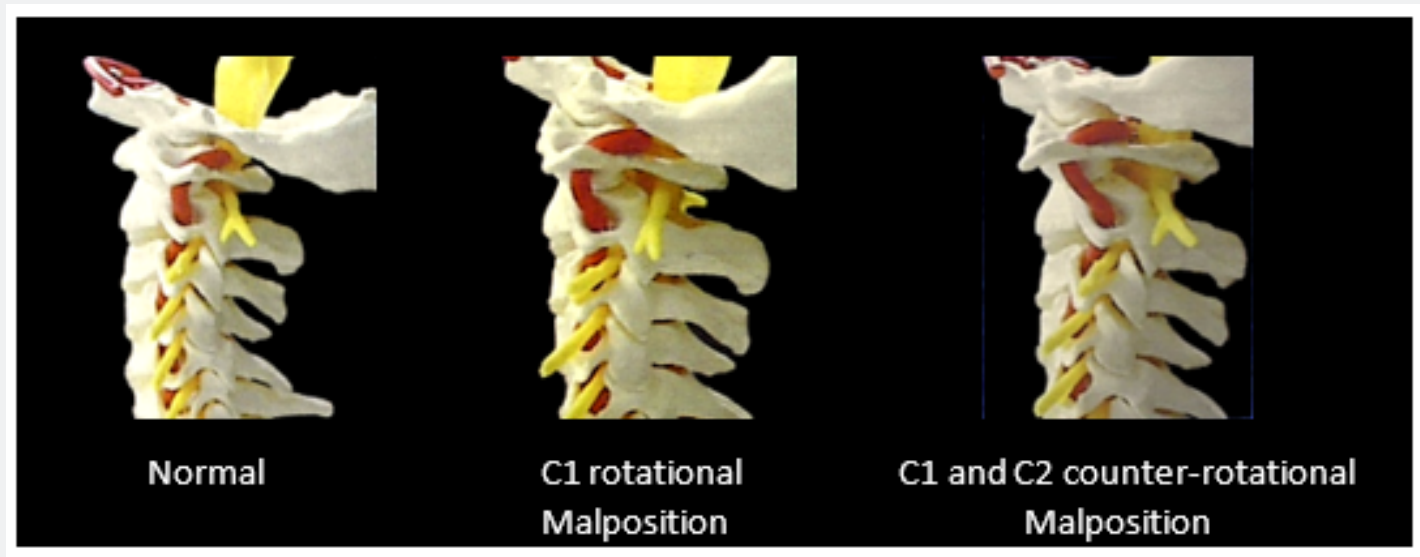

Figure 3 


\section{Vascular Effect - Venous}

The internal jugular vein (IJV) is the primary vascular drainage of the brain. It is relevant to recognize that the IJV descends just anterior to the $\mathrm{C} 1$ transverse process, and that compression of the IJV from the C1 vertebra can affect cerebral drainage capabilities of this vein and associated cranial hemodynamics.

Study: Incidence of Extrinsic Compression of the Internal Jugular Vein in Unselected Patients Undergoing CT Angiography American Journal of Neuroradiology. August 2012 M.V. Jaraman, J.L. Boxerman, et al. [2]. The study published in this edition of the American Journal of Neuroradiology noted that the internal jugular vein can become compressed by the atlas transverse process.

Study: Compression of the Internal Jugular Vein by the Transverse Process of the Atlas as the Cause of Cerebellar Hemorrhage After Supratentorial Craniotomy Eduardo Seoane, MD, Albert L Rhoton Jr., MD Surgical Neurology [3]. The study in this edition of Surgical Neurology concluded that the "obstruction of flow in the internal jugular vein at the site where the vein descends across the transverse process of the atlas is a likely cause of the venous hypertension that has resulted in the cerebellar hemorrhage reported in numerous cases after supratentorial craniotomy."

Study: Incidence and Distribution of Extravascular Compression of Extracranial Venous Pathway in Patients with Chronic Cerebrospinal Venous Insufficiency and Multiple Sclerosis Phlebology. 2014, Vol. 29(7) 476-479 Radak Djordje, Ilijevski Nenad, Kolar Jovo, Sagic Dragan, et al. [4]

"Our data indicate that extravascular compression of the extracranial venous pathway is frequent in multiple sclerosis patients with chronic cerebrospinal venous insufficiency, and that it is mainly due to compression caused by transverse processus of cervical vertebrae. Further studies are needed to evaluate potential clinical implications of this phenomenon." It is quite plausible and rational that an undetected and uncorrected craniocervical subluxation could negatively affect the vascular drainage of the brain, resulting in increased intracranial hypertension. This pressure increase could be a contributing factor in migraine headaches, dizziness, idiopathic seizures, TIAs, and other conditions. It seems plausible and rational that unresolving increased intracranial hypertension could complicate a patient's recovery from TBI and/or concussions, as well as CTE, and could be an initiator or exacerbator of PTSD, depression, and/ or other psychological conditions.

\section{Cerebrospinal Fluid (Csf) Flow}

Increased intracranial hypertension can have a disruptive effect on the cranial glymphatic system which can negatively alter the flow of the cerebrospinal fluid (CSF).

Study: The Glymphatic-Lymphatic Continuum: Opportunities for Osteopathic Manipulative Medicine Jama. 2016 Kyle
Hitscherich, OMS II; Kyle Smith, OMS II; Joshua A. Cuoco, MS, OMS II; et al. [5]. This study discusses the connection between the bloodbrain barrier, the CSF, the glymphatic system, and the potential for manipulative medicine to be used in the treatment of associated neurologic disorders.

Craniocervical subluxations that include rotational displacement of the $\mathrm{C} 1$ vertebra, $\mathrm{C} 2$ vertebra, both $\mathrm{C} 1$ and $\mathrm{C} 2$, and especially with counter-rotational displacement of the C1 and C2 vertebrae, can alter the space within the neural canal. It is plausible that diminished spinal canal diameter can cause a disruption of CSF flow, as has been noted in diagnostic imaging. It is plausible that reduced CSF flow due to craniocervical subluxation may negatively impact the potential for recovery from TBI, CTE, and concussions, as well as a potential contributor to the development of many brain-deterioration diseases.

\section{Elongated Styloid Process Impact}

Descending inferiorly from the skull are the styloid processes, the insertion points for the stylohyoid ligament. The styloid processes are non-compressible, boney structures just anterior to the IJV. Data analysis of over 3,000 patients from various EPIC clinics around the country revealed that, on average, $60 \%$ of patients over the age of 20 have styloid processes that are elongated down to the level of the $\mathrm{C} 1$ (atlas) transverse process. This creates an osseous structure both anterior and posterior to the IJV bilaterally, with the C1 transverse process posterior to the IJV. It is plausible that a rotationally misaligned C1 vertebra could result in vascular compression of the IJV into the elongated styloid, resulting in increased intracranial hypertension.

\section{Results}

Study: Incidence of Extrinsic Compression of the Internal Jugular Vein in Unselected Patients Undergoing CT Angiography M.V. Jayaraman, J.L. Boxerman, L.M. Davis, et al. American Journal of Neuroradiology [2].

Moderate stenosis was seen in $33.3 \%$ of right and $25.9 \%$ of left internal jugular veins. Severe stenosis was seen in $24.1 \%$ of right and $18.5 \%$ of left internal jugular veins." "The third parameter was the cause of the extrinsic compressions (styloid process, posterior belly of the digastric muscle, C1 transverse process). Examples are given in Figure 1. In all cases, the compression was caused by two factors. The posteromedial aspect of the compression was caused by the adjacent cervical vertebra, which provides a noncompressible structure against which the vein is pressed." We found that extrinsic compression of the internal jugular vein is common in unselected patients. The most common causes of this are the styloid process or posterior belly of the digastric muscle, often adjacent to the lateral mass of $\mathrm{C} 1$."

\section{Full Spine Effect}

A craniocervical subluxation can rapidly or gradually affect the entire spinal balance. Displaced upper cervical vertebrae, 


\section{Orthopedics and Rheumatology Open Access Journal (OROAJ)}

by altering the bilateral tonicity of the highly dense muscle spindles of the obliquus capitis inferioris muscles, can alter the mechanoreceptor feedback loops through the cerebellum to the vestibular nucleus. In response, the vestibular nucleus can induce a unilateral hyper-stimulation of the spinal posture muscles in different regions of the spine, or a hypo-stimulation of the opposite side. This imbalance of postural muscle tone can begin to bend the spine toward one side and/or create a functional "short leg" phenomenon as the pelvis becomes tilted. As the patient's vertical posture is now being altered, secondary full spine effects can occur, such as:
- $\quad$ Twisted rib cage

- $\quad$ Scoliosis

- Disc bulges/herniations

- $\quad$ Rotated sacrum

- Secondary vertebral malposition/fixation/subluxation (Figure 4)

Study: Experimental kyphoscoliosis induced in rats by selective brain stem damage
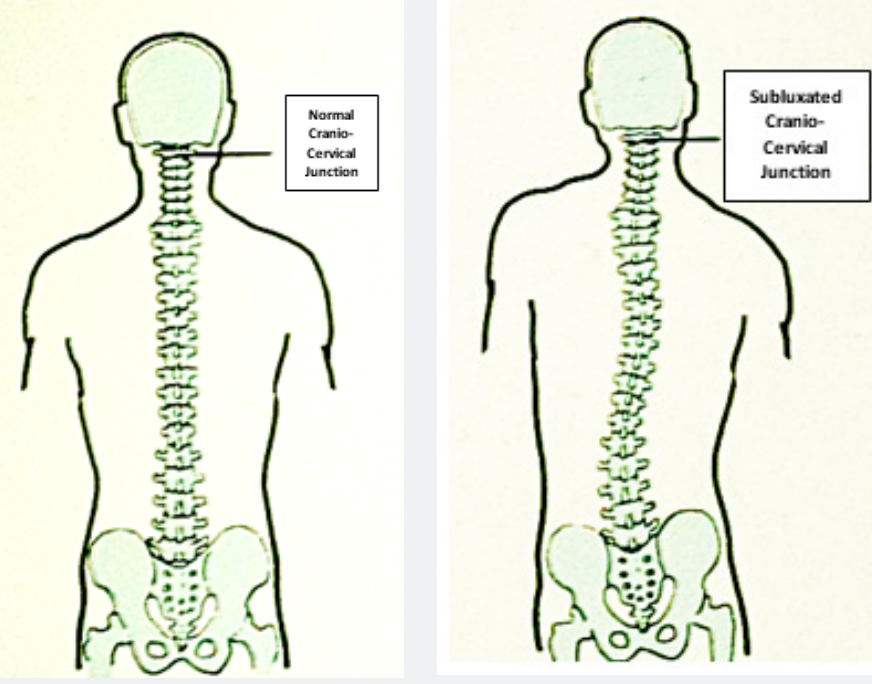

Figure 4

PMID: 1428313 C Barrios, J I Arrotegui. "The relationship between damage to particular brain stem nuclei related to postural control and equilibrium and the occurrence of spinal deformity has been investigated. The model used was the Wistar albino rat and the brain stem structures damaged were the gracillis nucleus, the superior colliculus and the lateral vestibular nucleus. Out of 60 brain stem damaged animals, 44 had accurate lesions from which 11 (25\%) showed kyphoscoliotic deformities. Rats with spinal deformity showed an imbalance of the paraspinal muscles when assessed by EMG; this was expressed by an increase of muscular activity on the convex side. This study indicates that postural dysfunction caused by brain stem damage may induce kyphoscoliosis in experimental animals and could be one of the underlying mechanisms in the production of human idiopathic scoliosis."

Study: The Vestibular-Evoked Postural Response of Adolescents with Idiopathic Scoliosis is Altered P M I D : 26580068 Jean-Philippe Pialasse, Martin Descarreaux, Pierre Mercier, et al. [6]. "Altered sensory reweighting of vestibular and proprioceptive information changed balance control of
AIS patients during and after vestibular stimulation. Therefore, scoliosis onset could be related to abnormal sensory reweighting, leading to altered sensorimotor processes.

\section{Lower Extremity Effect}

Craniocervical subluxation can result in a twisting of the body through altered neurostimulation of postural muscle tone, resulting in an altered center of gravity. This creates an imbalance of weight distribution on the lower extremities thereby increasing the demand on muscles, ligaments, and fascia. This can lead to unilateral soft tissue injury that occurs during a bilateral activity. Examples of this are unilateral plantar fasciitis, unilateral tendonitis, unilateral muscle strains and tears, a chronic ankle sprain, unilateral Achilles tendon injury, and unilateral OsgoodSchlatter disease (Figure 5). The longer a weight imbalance remains on the weight-bearing joints, the joints can deteriorate at a much faster rate. Examples of this include unilateral hip joint deterioration, unilateral knee joint deterioration, and unilateral ankle and foot pathologies. 


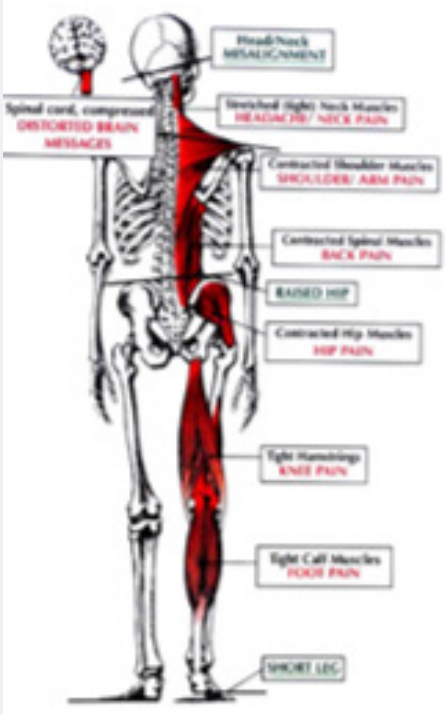

Muscular Imbalance

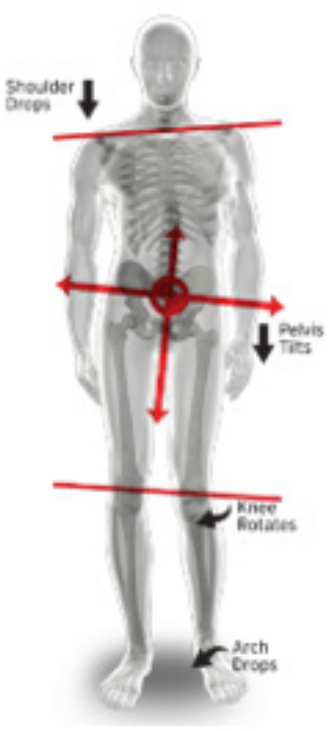

Weight Imbalance

Figure 5

\section{Craniocervical Structural Vulnerability}

The upper cervical spine is unlike any other vertebral segment of the body. Although vertebrae of the spine are stabilized by intervertebral discs and posterior interlocking facet joints, the transitionary occiput-C1-C2 region does not have these anatomical features. As a result, over $60 \%$ of cervical rotation occurs between $\mathrm{C} 1$ and $\mathrm{C} 2$, and the first 30 degrees of cervical flexion and extension occurs between occiput and C1. This area of the spine has the greatest range of motion, with minimal capacity for osseous fixation as the occiput-C1-C2 joints are a diarthrodial, synovial, freely moveable joint complex (Figure 6). Without the structural stabilizing components, the upper cervical spine is more vulnerable to injuries and malposition. It has been stated that there is no such thing as a head injury that does not also include a neck injury. When considering that the average weight of the adult head is $10-14$ lbs., there is substantially increased potential for the craniocervical junction to become subluxated during head or neck trauma, compared to any other, more stabilized joint of the spine. It is quite plausible that most head injuries would also include craniocervical joint injury. Varying directions and degrees of malposition are possible, causing associated dysfunctions and symptomatology.

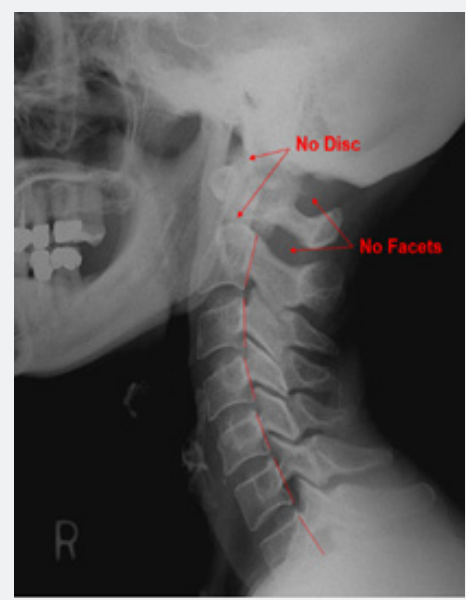

Figure 6 


\section{EPIC Examination}

The sequential neurophysical EPIC technique examination is as follows:

i. Supine leg length assessments are taken of each patient using a specific protocol to determine any global spinal imbalance that may be occurring due to the mechanoreceptor alteration of a craniocervical subluxation. This leg length assessment is taken with the patient laying supine with their head supported by a wedged pillow, to maintain spinal postural curves more consistent with upright posture.

ii. While still in the supine position, the patient's lumbar myotonicity is manually evaluated for bilateral balance or lack thereof. In this position the postural muscles should be deactivated, and any unilateral hypertonicity may be indicative of abnormal neurostimulation to the musculature.

iii. With the patient still in the supine position, an isolated shoulder strength test is performed using a modified deltoid break test, bilaterally for comparison. Strength imbalance is noted.

iv. Remaining in the supine position, a craniocervical syndrome test is performed as examination steps 1-3 are performed again to observe any functional changes. v. With patient now seated, ganglion compartment syndrome assessment is performed to assess for potential edematous accumulation from a craniocervical subluxation.

vi. Overall patient posture is observed.

vii. Fakuda's step test is then used to ascertain any functional loss of center of gravity or cerebellar functional irregularities.

A combination of these findings is used to confirm the presence or absence of neurological indicators of a craniocervical subluxation. Additional tests may also be used, when indicated, to confirm the extent of dysfunction and pathological progression. If the indicators are present, EPIC protocol requires imaging.

\section{EPIC Radiographs}

The EPIC technique protocol currently utilizes digital radiographic imaging. The EPIC digital x-ray protocol obtains a minimum of three images of a patient. The name of these three radiographs is congruent with the planes of reference they are attempting to capture: Sagittal, Frontal, and Horizontal x-rays. A fourth $\mathrm{x}$-ray view may be taken to accurately visualize the surface of the C2 vertebra on its own plane of reference. That film is termed the Axial x-ray (Figure 7).

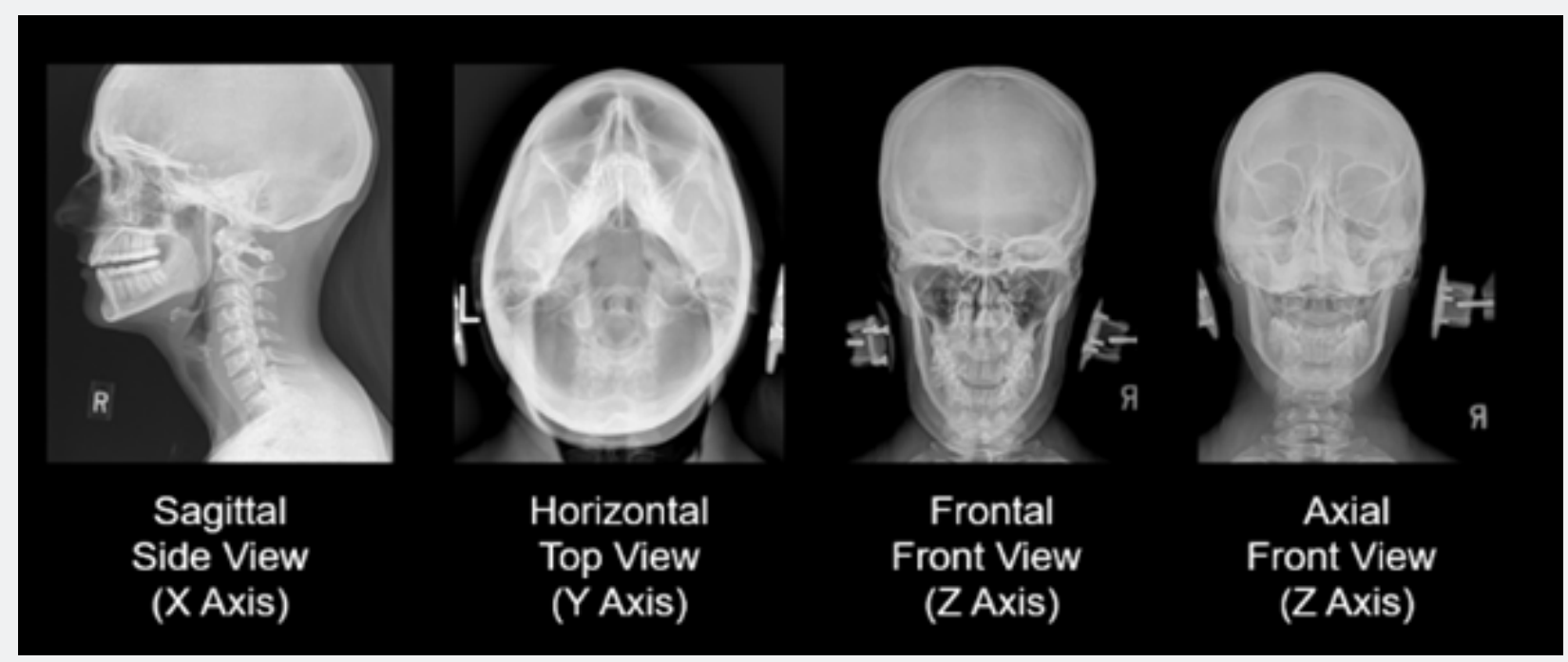

Figure 7

\section{EPIC Radiographic Analysis}

The EPIC radiographic analysis uses computer software programs consisting of physics and engineering mathematic formulas applied through contrast differentiation, multi-planar line assessment, dimensional transposition, and CAD overlays designed to assist the doctor with obtaining accurate calculations.
Genetic malformations and structural misalignments are measured for each patient. This is imperative, as it was noted by research in 2009 that traditional x-ray analysis for structural malposition can be complicated by genetic asymmetry.

Study: Asymmetry in atlas bone specimens: a pilot study using radiographic analysis JCM 2009: 8, 72-76 John Hart, Matt 


\section{Orthopedics and Rheumatology Open Access Journal (OROAJ)}

Christopher, Ralph Boone [7]. "These 10 specimens showed an average difference of $0.95 \mathrm{~mm}+/-1 \mathrm{SD}(0.67 \mathrm{~mm})$ or 2 SDs $(1.34 \mathrm{~mm})$ between the left and right sides of the atlas vertebrae. Differences found on radiographs may be due to asymmetry and not actual misalignment."

The EPIC radiographic analysis currently evaluates for 23 genetic asymmetries and/or anomalies to determine what a patient's "normal" structural position should be. The EPIC analysis additionally measures four multi-directional misalignments through computerized analysis to determine the biomechanics of vertebral misalignment, the degree-specific line of drive for the adjusting trajectory, and the targeting location for the impulse.

The four multi-directional misalignments measured are:

- C1 (atlas) to occiput rotational malposition around the $\mathrm{Z}$ axis $(\varnothing \mathrm{Z})$

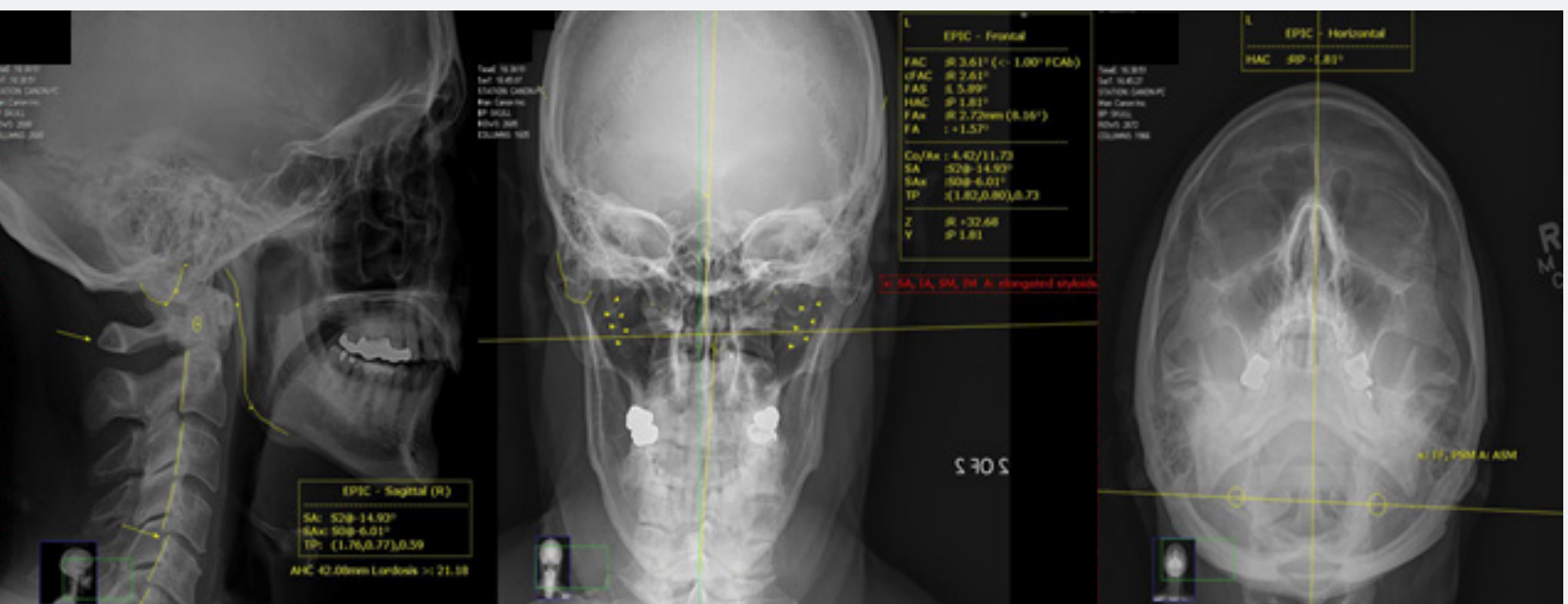

Figure 8

\section{The Adjusting Difference}

Most chiropractic adjustments or spinal manipulations have a relatively general application that is administered along the angle of the facet joints to release the fixation of the segment. In that the subluxated C1 and C2 vertebrae do not experience an osseous fixation, as they do not have interlocking posterior facet joints, an alignment procedure for the craniocervical junction does not require a focus on restoring normal motion, but rather correct alignment. The $\mathrm{C} 1$ vertebra can misalign around the convexly curved occipital condyles of the bottom of the skull and can misalign around the convexly curved C2 superior surface. Systems of analysis typically include a consideration of the convexity of the surfaces for customizing an accurate angle for each individual patient's correction. Also, there are four multi-directional, multinumerical misalignments of the craniocervical junction as well as numerous genetic asymmetries in the anatomical development of the bones that must be measured and accounted for in customizing the adjusting procedure (Figure 9). Researchers
- C1 (atlas) to cervical spine rotational malposition around the $\mathrm{Z}$ axis (ØZ)

- C1 (atlas) rotational malposition around the Y axis (ØY)

- C2 (axis) rotational malposition around the Y axis (ØY)

Due to the fact that the analysis coordinate measurements are within $1 / 100^{\text {th }}$ of a degree, the accuracy of analysis directly equates to a decreased potential for error in the adjusting angle. With increased accuracy in the adjusting angle and targeting of the impulse, the amount of force used to accomplish the adjustment can be minimized. This accuracy also equates to only requiring a single impulse for the correction (Figure 8). The radiographic analysis also determines the biomechanical influences necessary for positioning the patient prior to the adjustment in order to minimize joint resistance and influence the four misalignments to reduce proportionately and equally with a single, correctional impulse.
White and Panjabi noted that the $\mathrm{C} 1$ vertebra can misalign with a maximum lateral displacement of only 5 degrees, which is $1 / 8$ inch [8]. Combining this fact along with the lack of joint fixation concludes that aligning the craniocervical junction may only require minimal force and minimal depth. Also, contrary to most adjustments and/or manipulations that might take a vertebral segment to its full end range of motion, and then thrust beyond to perform the treatment, adjustments of the craniocervical junction can be done in normal range of motion, protecting the integrity of the surrounding soft tissue.

\section{The Adjusting Technology}

The EPIC procedure aligns the craniocervical junction using a percussive sound wave impulse, rendered on a precisely calibrated line of drive (angle) that is accurately targeted to the $\mathrm{C} 1$ (atlas) transverse process.

This is done using the Integrity Genesis adjusting instrument. This device is the first 3 -axis percussion adjusting instrument in 
the history of chiropractic. It is a table-mounted instrument that is FDA registered, patent-pending, manufactured under ISO 90012015 manufacturing standards, and is exclusive to EPIC. The design of the Integrity Genesis allows for coordinate calibration in the axis of adjusting to be consistent with the axis of misalignment, and the coordinate settings for the instrument are calibrated to the exact leverage angle measured on the x-rays based upon the misalignments and genetic asymmetries/anomalies (Figure 10). Digital calibration, laser targeting, motorized shoulder piece, dual patient safety mechanisms, airplane-grade materials, medicalgrade high-capacity linear rails and reciprocating bearings, solid state Teflon, IPX6 protection class electronics, patented construction systems and more are innovative features of the Integrity Genesis.

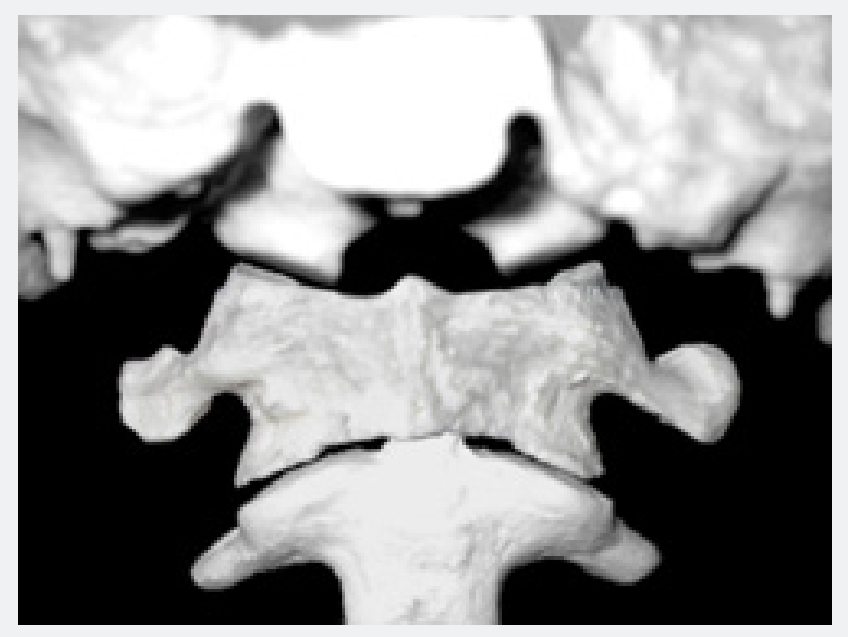

Figure 9
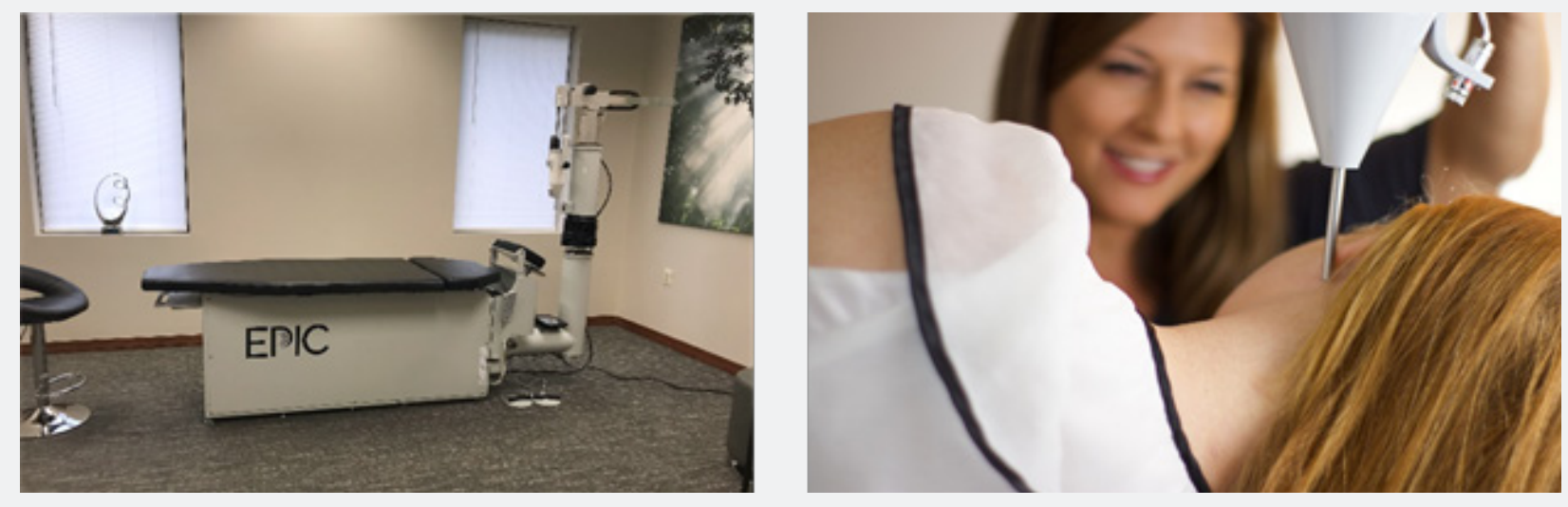

Figure 10

\section{The Adjusting Force}

The use of sound wave therapy has become more common in healthcare. Lithotripsy for breaking up kidney stones, ultrasound used to treat muscle and joint injury, radiofrequency ablation of nerves, and even the sound wave treatment of cancer, are just a few examples of current uses of sound treatments. The use of a percussive impulse adjusting to alter the $\mathrm{C} 1$ vertebral alignment has been utilized in the chiropractic profession since the 1970s. The EPIC procedure uses a percussive impulse generated by an electromagnetic striking device, to deliver a gentle, non-invasive, degree-specific force at a specified spinal location. The device is placed just off the skin, calibrated on a multi-axis coordinate setting, and aligned to the patient's C1 transverse process. All patient positioning for the adjustment, as well as all instrument calibrating systems, are customized for each patient based upon the radiographic measurements obtained through the computerized analysis. Using mathematics, physics, and technology, the EPIC doctor is able to rely less on opinion and experience, and more on measurements and science to render precision care for a patient.

Additionally, the use of a percussive impulse can overcome anatomical variables that can complicate typical adjustments or spinal manipulations. Basilar invagination, overgrown mastoids, elongated styloid processes, ear cartilage, large mandibles, etc. can interfere with being able to send a manual, excursion, or 
"pushing" type of adjusting force into the contact point of the C1 transverse process, which is located typically $3 / 4-1$ inch below the surface of the skin. With the use of a percussive impulse, the frequency can transmit through these anatomical obstacles to affect the $\mathrm{C} 1$ transverse process.

\section{A Gentle Approach}

For over 125 years, chiropractic adjustments have been performed with varying amounts of force. Some struggle to comprehend how an EPIC sound wave is enough force to change the craniocervical alignment. Here are reasons why the EPIC adjustment can be so gentle:

- $\quad$ Patient is in a side-lying position; therefore, gravity is not counteracting the adjusting force.

- $\quad$ With patient in a side-lying position, the 10-14lb skull weight is not pressing down into the joints.

- $\quad$ Patient is positioned to immobilize the skull so that C1 can easily move around the adjoined occipital condyles.

- $\quad$ Patient is positioned in a unique, customized manner to reduce resistance within the craniocervical joints.

- Patient does not subconsciously guard for the adjustment, as it occurs in normal range of motion, without movement of the patient's head or neck, and without any physical force.

Patient also does not guard against the treatment because there is no pain with an EPIC adjustment.

- The adjusting line of drive is customized to the exact and unique coordinate measured for each patient based on their genetic asymmetries, anomalies and misalignments, thereby increasing accuracy which requires less force to move the anatomy.

\section{Evidence-Based Outcomes}

Immediately following the EPIC treatment, the neurophysiology begins to change. Evidence of this change be the patient's body begins untwisting, the low back myotonicity begins to normalize, and the leg length begins to balance. Subsequently, the posture begins normalizing, the head and neck begin to straighten in relation to the body, the lateral pelvic shift improves, and the biomechanics of the weight-bearing joints begin to improve (Figure 11). Every patient is post x-rayed immediately after their first EPIC adjustment. These x-rays are taken with the same procedures as the original and are measured in the same method of analysis. The new alignment is assessed to determine the effectiveness of the adjustment and adequacy of structural repositioning (Figure 12).

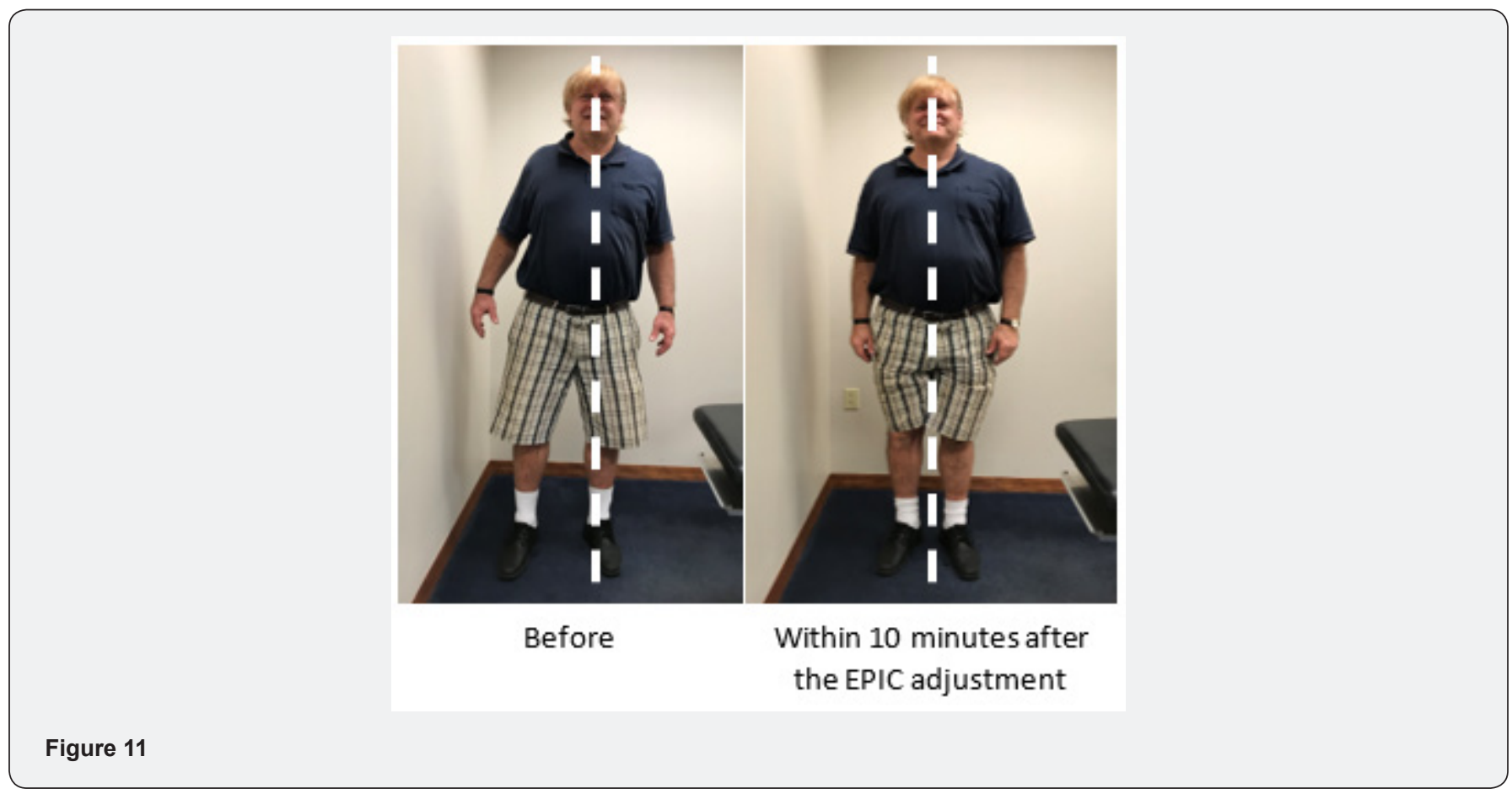




\section{Orthopedics and Rheumatology Open Access Journal (OROAJ)}

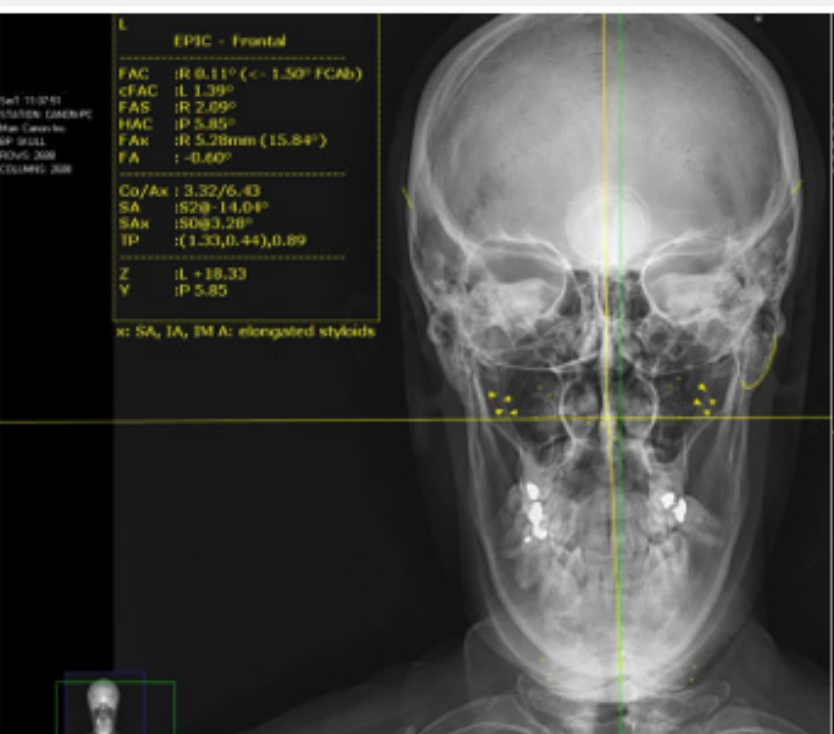

Pre-adjustment Frontal x-ray on 12/28/20

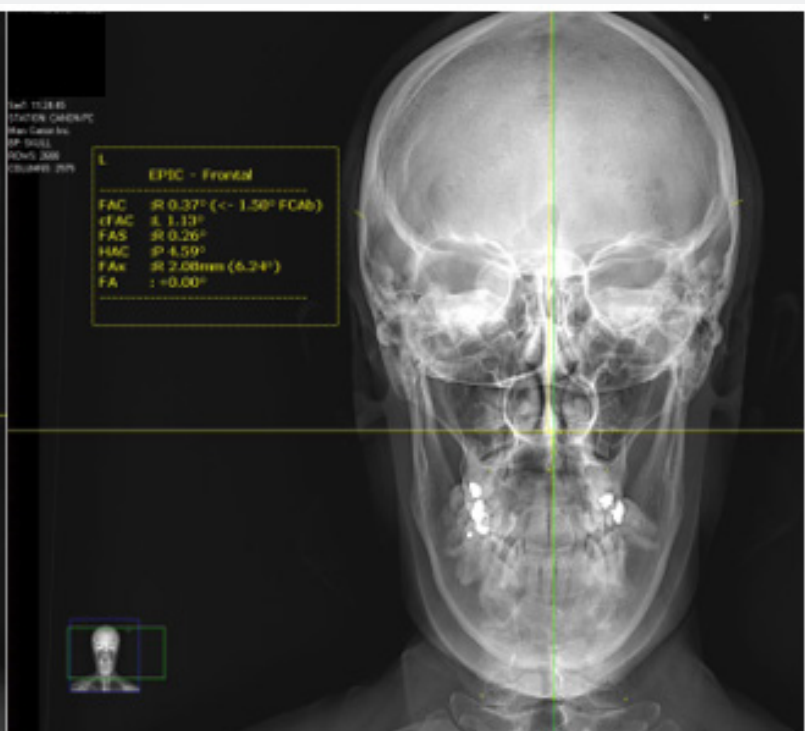

Post-adjustment Frontal x-ray on 12/29/20

Figure 12

Figure 13

These before and after measurements are entered into EPIC's software program called Sonus Blueprint. This program quantifies and compares all structural relationships to assess the adequacy of misalignment reduction with the EPIC adjustment. From the post x-ray interpretation, Sonus Blueprint will help guide the doctor on what modifications need to be made, if any, in future adjustments of that patient to obtain an even better correction. Each doctors' results are tracked and compared with those results of other EPIC practitioners. From these results, EPIC is able to ascertain a measurable standard of care by which all doctors practicing EPIC can be held accountable. EPIC is also able to introspect the outcomes to determine refinements in the protocols of care inside the EPIC technique. Sonus Blueprint also tracks demographics, chief complaints, neurophysical examination findings, radiographic structural findings, genetic asymmetries, and much more. This information can be cross correlated on an international platform for seemingly limitless research studies and can easily be compared with additional patient outcome assessments (Figure 13). 


\section{Holding}

The EPIC spinal alignment procedure is expected to correct the craniocervical subluxation with the very first treatment. Variables that could affect how long the alignment will remain in proper position include:

- time necessary for soft tissue morphology around the new structural alignment

- patient not protecting their craniocervical junction following the procedure

- $\quad$ patient experiencing a trauma

However, the EPIC procedure has four unique benefits in its application that tend to allow the alignment to "hold" for exponentially longer periods of time compared to traditional chiropractic care. These benefits include:

- the correction removes all four misalignments simultaneously and instantaneously

- the correction aligns a patient into their genetically normal position

- the correction improves/restores the vertical center of gravity of the skull over the neck

- $\quad$ the correction preserves the integrity of the ligamentous support system by maintaining all adjusting force applications to be performed within a patient's normal range of motion.

Due to these factors, patients tend to maintain ("hold") the EPIC spinal correction for longer and longer periods of time compared to traditional spinal manipulative care. EPIC is a chiropractic procedure that should be viewed as a corrective adjusting procedure that works to provide longevity of positive patient outcomes, rather than as a short-term relief therapy. As the craniocervical alignment stabilizes and holds its correct position for longer periods of time, mechanoreceptor feedback loops can balance, postural erector muscles can then balance, the full spine has the opportunity to untwist and center, which can have a significant positive effect on intervertebral spinal discs and weight bearing joints as they regain their biomechanical stability.

\section{Collaboration}

Although the EPIC procedure has a powerful stand-alone value, it has a unique ability to integrate well with most other supportive treatments. EPIC is not intended to be used to compete with other chiropractic or medical procedures, but rather to comanage cases for the sake of the patient. There are significant potential benefits to other physicians co-managing/co-treating cases with EPIC.

Benefits in the areas of diagnostic accuracy and treatment outcomes include, but are not limited to:

\section{Diagnostic accuracy}

- Neurologic assessments taken caudal to the craniocervical region could have a greater diagnostic accuracy without the neurological irregularities caused by the craniocervical subluxation.

- Vascular assessments for intracranial blood flow could potentially be more accurate without altered hemodynamics of the vertebral artery or internal jugular vein associated with a craniocervical subluxation.

Imaging studies assessing the cerebrospinal fluid flow and associated intracranial pressure levels could be more accurate without the potential CSF disruptive sequelae of a craniocervical subluxation.

Vascular assessments to screen for, and causation determination of, systemic high blood pressure or secondary vascular restrictions may be more accurate in the absence of a craniocervical subluxation.

\section{Treatment efficacy}

With an untwisting spine due to improved bilateral spinal muscle balance from EPIC craniocervical care, secondary spinal adjusting procedures used to treat other full spine considerations may be even more effective. Secondary spinal misalignments may correct with less overall resistance and may have an increased propensity to maintain proper alignment as well.

Any muscular treatments (i.e. massage therapy, neuromuscular therapy, physical therapy) for hypertonicity/ spasm may show a significant improvement in response to treatment as the mechanoreceptor balance to those muscles improves with maintaining proper craniocervical alignment. Muscle tone is controlled by nerves, and nerves are protected by proper alignment of the spine.

As the spine uprights with an improving vertical center of gravity on the circular fibers and nucleus of the spinal disc, disc treatments designed to address disc bulge and/or herniation may demonstrate a significantly more favorable response following the use of EPIC.

- Physical therapy attempting to rehabilitate the stabilizing muscle groups around a joint may discover patients responding more quickly to the therapy, if the neuro-stimulatory control of the associated muscles is unobstructed due to adequate craniocervical alignment. Additionally, it is logical to assume that physical therapy for improving the health and integrity of weight bearing joints should respond more favorably with equal, bilateral weight distribution to the lower extremities and balanced posture associated with proper craniocervical alignment. 
- Vascular treatments designed to improve intracranial blood flow should be more effective without the hemodynamic disruptions associated with a craniocervical subluxation.

\section{Conclusion}

EPIC is a chiropractic technique that utilizes neurology, physics, engineering and technology to analyze and correct the craniocervical alignment. Accurate alignment of the craniocervical junction can positively affect many neurovascular and biomechanical functions throughout the body, and EPIC is therefore a logical assessment and treatment option to help improve patient outcomes with many diverse conditions. It is plausible that EPIC can exist in any healthcare setting and is able to effectively co-manage cases with parallel treatment models. As EPIC is focused on the use of progressing technology, positive patient outcomes, and a measurable standard of care, the EPIC technique is advancing the chiropractic profession forward into the evidence-based model of care that the healthcare community is looking for and that the public deserves. As with all research, more studies are needed comparing this method with others, as well as the corresponding effect of neurovascular improvements on various conditions.

\section{References}

1. G Bakris, M Dickholtz Sr, P M Meyer, G Kravitz, E Avery, et al. (2007) Atlas vertebra realignment and achievement of arterial pressure goal in hypertensive patients: a pilot study. J Hum Hypertens 21(5): 347352.
2. M V Jayaraman, J L Boxerman, L M Davis, R A Haas, J M Rogg (2012) Incidence of extrinsic compression of the internal jugular vein in unselected patients undergoing CT angiography. AJNR Am J Neuroradiol 33(7): 1247-1250

3. Eduardo Seoane, Albert L Rhoton (1999) Compression of the internal jugular vein by the transverse process of the atlas as the cause of cerebellar hemorrhage after supratentorial craniotomy. Surg Neurol 51(5): 500-505.

4. Rakad Djordje, Ilijevski Nenad, Kolar Jovo, Sagic Dragan, Antonic Zelimir, et al. (2014) Incidence and distribution of extravascular compression of extracranial venous pathway in patients with chronic cerebrospinal venous insufficiency and multiple sclerosis. Phlebology 29(7): 476-479.

5. Kyle Hitscherich, OMS II, Kyle Smith, OMS II, Joshua A, et al. (2016) The glymphatic-lymphatic continuum: Opportunities for osteopathic manipulative medicine. Jama 116(3): 170-177.

6. Jean-Phillippe Pialasse, Martin Descarreaux, Pierre Mercier, Jean Blouin, Martin Simoneau, et al. (2015) The vestibular-evoked postural response of adolescents with idiopathic scoliosis is altered. PLoS ONE. November 10(11): e0143124.

7. John Hart, Matt Christopher, Ralph Boone (2009) Asymmetry in atlas bone specimens: a pilot study using radiographic analysis. Journal of Chiropractic Medicine 8(2): 72-76.

8. Panjabi M, Dvorak J, Duranceau J, Yamamoto I, Gerber M, et al. (1988) Three-dimensional movements of the upper cervical spine. Spine. 13(7): 726-730.

\section{Your next submission with Juniper Publishers will reach you the below assets}

- Quality Editorial service

- Swift Peer Review

- Reprints availability

- E-prints Service

- Manuscript Podcast for convenient understanding

- Global attainment for your research

- Manuscript accessibility in different formats

( Pdf, E-pub, Full Text, Audio)

- Unceasing customer service

Track the below URL for one-step submission https://juniperpublishers.com/online-submission.php 